Article

\title{
Design, Synthesis and Anti-Tobacco Mosaic Virus (TMV) Activity of 5-Chloro- $N$-(4-cyano-1-aryl-1H-pyrazol-5-yl)-1-aryl- 3-methyl-1H-pyrazole-4-carboxamide Derivatives
}

\author{
Jin-Jing Xiao ${ }^{1, \dagger}$, Min Liao ${ }^{1, \dagger}$, Ming-Jie Chu ${ }^{1}$, Zi-Li Ren ${ }^{1}$, Xin Zhang ${ }^{2}$, Xian-Hai Lv ${ }^{1, *}$ and \\ Hai-Qun Cao ${ }^{1, *}$
}

1 College of Plant Protection, Anhui Agricultural University, Hefei 230036, China;

E-Mails: xiaojj187012@163.com (J.-J.X.); liaomin3119@126.com (M.L.); chumingjie@ahau.edu.cn (M.-J.C.); renzilix@163.com (Z.-L.R.)

2 State Key Laboratory of Pharmaceutical Biotechnology, Nanjing University, Nanjing 210093, China; E-Mail: zhangxinzxn@126.com

$\dagger$ These authors contributed equally to this work.

* Authors to whom correspondence should be addressed; E-Mails: 1vxianhai@ahau.edu.cn (X.-H.L.); haiquncao@163.com (H.-Q.C.); Tel./Fax: +86-551-6578-6906 (X.-H.L.).

Academic Editor: Derek J. McPhee

Received: 28 October 2014 / Accepted: 29 December 2014 / Published: 7 January 2015

\begin{abstract}
A series of novel pyrazole amide derivatives 3a-3p which take TMV PC protein as the target has been designed and synthesized by the reactions of 5-chloro-1-aryl-3-methyl$1 H$-pyrazole-4-carboxylic acids with 5-amino-1-aryl-1H-pyrazole-4-carbonitriles. All the compounds were characterized by ${ }^{1} \mathrm{H}-\mathrm{NMR}$, mass spectroscopy and elemental analysis. Preliminary bioassays indicated that all the compounds acted against the tobacco mosaic virus (TMV) with different in vivo and in vitro modes at $500 \mu \mathrm{g} / \mathrm{mL}$ and were found to possess promising activity. Especially, compound 3p showed the most potent biological activity against tobacco mosaic virus (TMV) compared to ningnanmycin, and a molecular docking study was performed and the binding model revealed that the pyrazole amide moiety was tightly embedded in the binding sites of TMV PC (PDB code: 2OM3).
\end{abstract}

Keywords: pyrazole amide; synthesis; anti-TMV; molecule docking 


\section{Introduction}

Tobacco mosaic virus (TMV) is one of the most extensively studied among a number of plant viruses. Its infections are very widely distributed [1-3] and can cause serious damage and large economic losses. TMV is a growing problem in the contemporary pesticide field, as only a few antiviral agents are currently available in practice, and almost no treatment can control TMV effectively [4], so the identification of new antiviral agent with novel mechanisms of action is critically needed to overcome this viral infections.

Recently, different targets essential to plant viruses that could be new weapons to design and discover new antiviral drugs have been studied and validated. Most antiviral drugs exhibit their activities through interaction with viral proteins $[5,6]$. Coat protein-mediated resistance to TMV involves mainly two independent mechanisms: (i) interference by transgenic CP with disassembly of challenge virus; and (ii) interference of transgenic $\mathrm{CP}$ with formation of replication complexes, thus interfering with virus movement [7]. CP is an important part of the genomic structure of the tobacco mosaic virus (TMV), and its main function not only includes the protection of DNA from degradation, but it also has a close relationship with the long-distance movement of TMV and host symptoms [8]. Therefore, coat protein (PC) is usually used as a molecular target for drug action, and the study of TMV CP mainly focuses on $\mathrm{CP}$-mediated resistance, interference the polymerization of $\mathrm{CP}$ by agents and so on. Many small molecule ligands. including pyrazole derivatives, that combine with molecular targets have been reported [9-12]. Inspired by the above notion, we hope to design a new compound which is able to target TMV PC to inhibit viral assembly through small molecule-coat protein interactions.

Pyrazole derivatives have great importance in the medicinal and pesticide fields [13-15], due to their broad range of biological activity [16-18]. The molecules of many modern drugs with antiphlogistic, antidiabetic, analgesic, and acaricidal activity in medicine [19] and herbicidal [20-22], antibacterial [23-25], acaricidal and insecticide [26,27] in the pesticide area contain pyrazole rings as a structural fragment. As shown in Figure 1, several pyrazole derivative products have been launched or announced, and they all contain several common structural features that are essential to their activity, including an amide bond, pyrazole and an aromatic ring attached to the carbonyl of the amide bond.
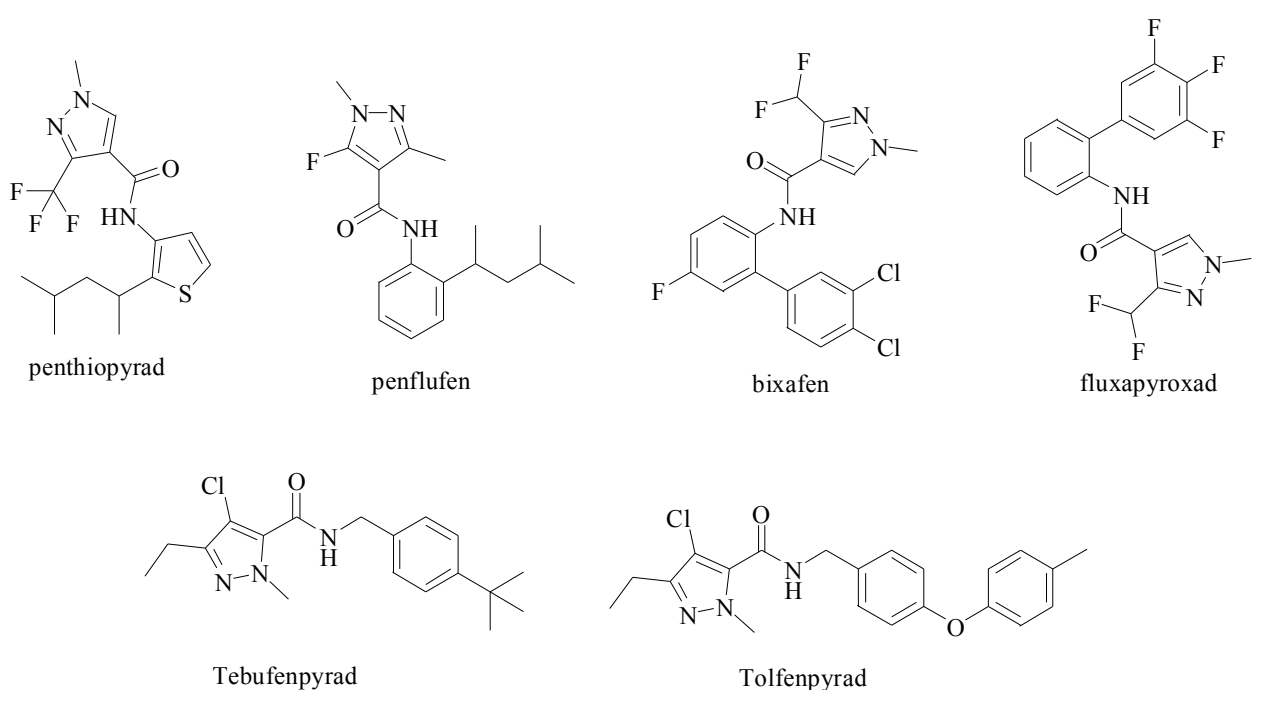

Figure 1. Pyrazole derivative products. 
Modification of the structural profile by the change of substituents at the 1-, 3-, or 4-positions in the pyrazole ring can bring about significant changes in bioactivity [28], and linking the pyrazole group with a structurally diverse side chain is an effective way to obtain new heterocyclic derivatives with high antiviral activities [29]. We assumed that if nitrile-containing pyrazole pharmacophores were introduced into the pyrazole scaffold, thus transforming the structure reported by Ouyang et al. [30], the resulting compounds may be interesting lead structures for antiviral agent development (Figure 2), because the nitrile can modulate the physicochemical and pharmacokinetic properties to improve bioavailability, enhance the selectivity and binding affinity to target proteins by hydrogen bond interactions, covalent interactions, polar interactions, and $\pi-\pi$ interactions [31-33], and cyano substitution in the design of drugs has become one of the important research strategies in optimizing the structure of lead compounds [34].

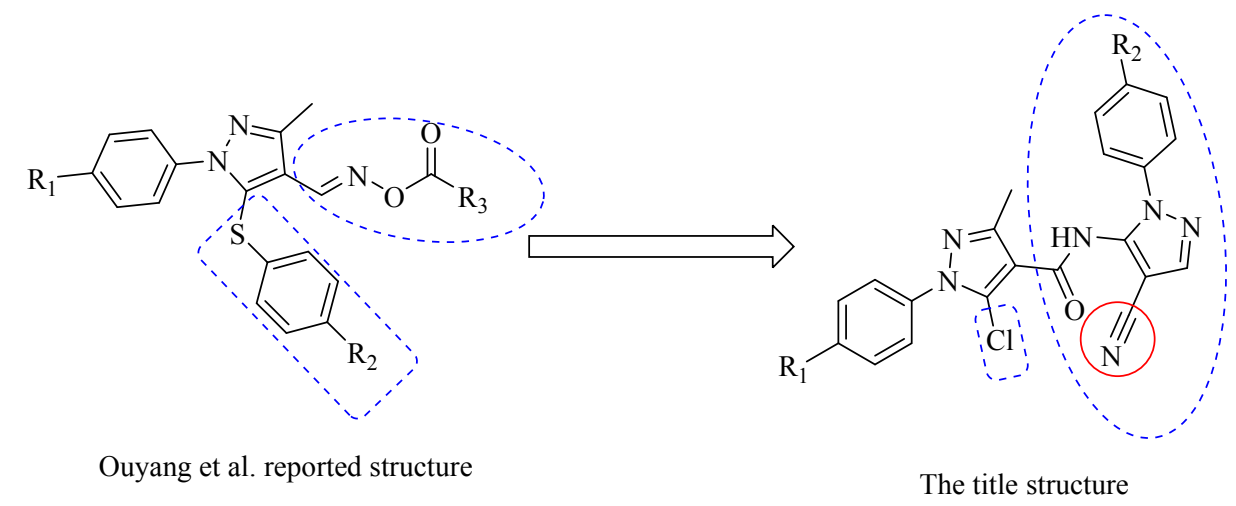

Figure 2. Design of the target compounds.

We describe herein the synthesis and antiviral activity of a series of new pyrazole amide derivatives (Figure 3). Molecular docking was performed and the binding model revealed that the title pyrazole amide was tightly embeded in the binding sites of TMV PC (PDB code: 2OM3) (Figure 4). The anti-TMV activities of these compounds were subsequently evaluated, and a structure-activity relationship was established in terms of their anti-TMV activity.

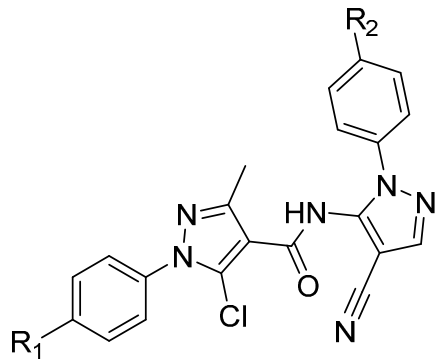

\begin{tabular}{ccc}
\hline Compound NO. & $\mathbf{R}_{\mathbf{1}}$ & $\mathbf{R}_{\mathbf{2}}$ \\
\hline $\mathbf{3 a}$ & $\mathrm{H}$ & $\mathrm{H}$ \\
$\mathbf{3 b}$ & $\mathrm{H}$ & $\mathrm{Cl}$ \\
$\mathbf{3 c}$ & $\mathrm{H}$ & $\mathrm{CH}_{3}$ \\
$\mathbf{3 d}$ & $\mathrm{H}$ & $\mathrm{F}$ \\
$\mathbf{3 e}$ & $\mathrm{Cl}$ & $\mathrm{H}$ \\
\hline
\end{tabular}

Figure 3. Cont. 


\begin{tabular}{ccc}
\hline Compound NO. & $\mathbf{R}_{\mathbf{1}}$ & $\mathbf{R}_{\mathbf{2}}$ \\
\hline $\mathbf{3 f}$ & $\mathrm{Cl}$ & $\mathrm{Cl}$ \\
$\mathbf{3 g}$ & $\mathrm{Cl}$ & $\mathrm{CH}_{3}$ \\
$\mathbf{3 h}$ & $\mathrm{Cl}$ & $\mathrm{F}$ \\
$\mathbf{3 i}$ & $\mathrm{CH}_{3}$ & $\mathrm{H}$ \\
$\mathbf{3 j}$ & $\mathrm{CH}_{3}$ & $\mathrm{Cl}$ \\
$\mathbf{3} \mathbf{k}$ & $\mathrm{CH}_{3}$ & $\mathrm{CH}_{3}$ \\
$\mathbf{3}$ & $\mathrm{CH}_{3}$ & $\mathrm{~F}$ \\
$\mathbf{3} \mathbf{m}$ & $\mathrm{F}$ & $\mathrm{H}$ \\
$\mathbf{3 n}$ & $\mathrm{F}$ & $\mathrm{Cl}$ \\
$\mathbf{3 0}$ & $\mathrm{F}$ & $\mathrm{CH}_{3}$ \\
$\mathbf{3 p}$ & $\mathrm{F}$ & $\mathrm{F}$ \\
\hline
\end{tabular}

Figure 3. The structure of compounds 3a-3p.

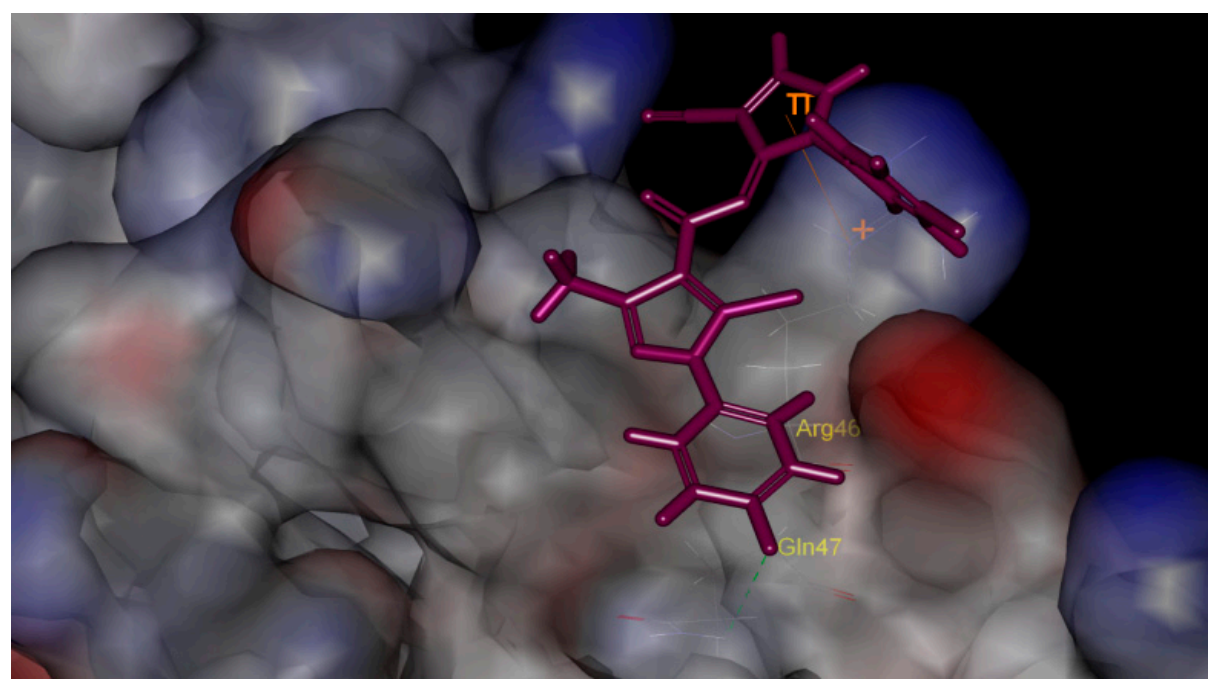

Figure 4. Docking model of compound $3 p$ with TMV PC. It is nicely bound to the TMV PC one H-bond (GIn47-F:2.49 $\AA, 109.02^{\circ}$ ) and has a $\pi$-cation interaction (4.65 $\AA$ ).

\section{Results and Discussion}

\subsection{Chemistry}

The synthetic route is shown in Schemes 1-3. Compounds 1a-1d were prepared in accordance with the literature procedure [35] from the reactions of substituted phenylhydrazines with ethyl acetoacetate in anhydrous ethanol medium. Then the reaction mixtures was added to a cold solution of DMF and $\mathrm{POCl}_{3}$. Finally, the products were oxidized with $\mathrm{KMnO}_{4}$, to give the 5-chloro-1-aryl-3-methyl- $1 H$ pyrazole-4-carboxylic acids 1 (Scheme 1).

Compounds 2a-2d were obtained from substituted phenyl hydrazine hydrochlorides and 2-(ethoxymethylene) malononitrile in ethanol medium, which was refluxed for $3 \mathrm{~h}$ to produce the 5-amino-1-aryl-1H-pyrazole-4-carbonitriles 2a-2d [36] (Scheme 2). 


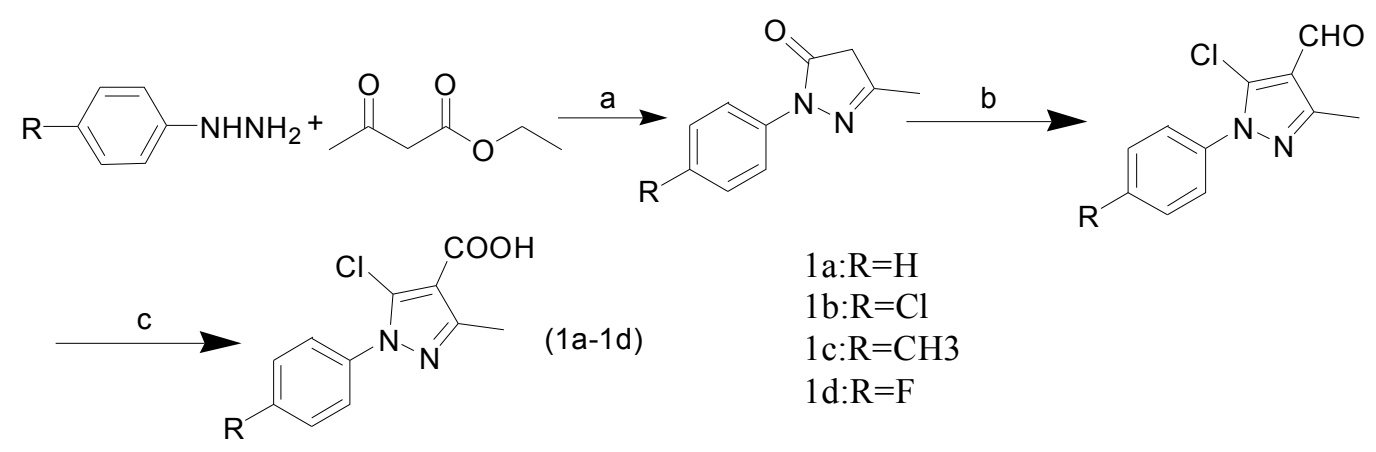

Scheme 1. General synthesis of compounds 1a-1d. (a) $\mathrm{H}_{2} \mathrm{O}$, ethanol, $60{ }^{\circ} \mathrm{C}$, TLC; (b) DMF, $\mathrm{POCl}_{3}, 80-85{ }^{\circ} \mathrm{C}, \mathrm{TLC} ;$ (c) $\mathrm{KMnO}_{4}, 70-80{ }^{\circ} \mathrm{C}, \mathrm{TLC}$.

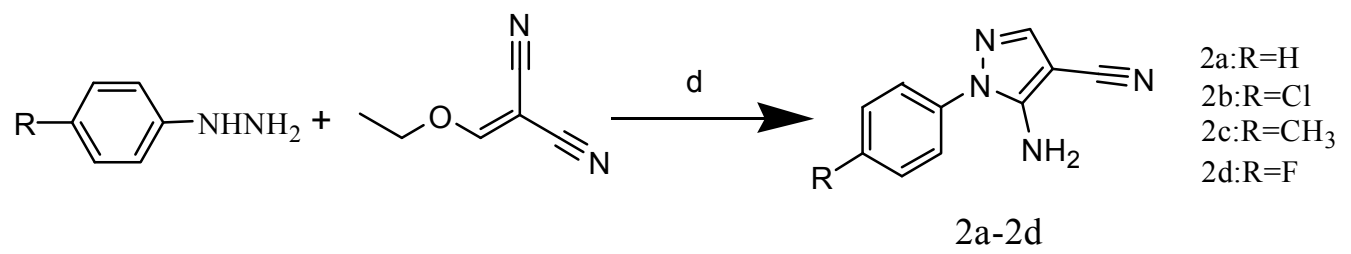

Scheme 2. General synthesis of compounds $\mathbf{2 a}-\mathbf{2 d}$. (d) $\mathrm{H}_{2} \mathrm{O}, \mathrm{NaOH}$, ethanol, 3 h, reflux.

The compounds 1 and 2 were reacted using 1-ethyl-3-(3-dimethylaminopropyl) carbodiimide hydrochloride (EDCI) and N-hydroxybenzotriazole (HOBt) in DMF medium in the presence of triethylamine as catalyst at room temperature to produce the 5-chloro-N-(4-cyano-1-aryl-1H-pyrazol-5yl)-1-aryl-3-methyl-1H-pyrazole-4-carboxamides 3a-3p (Scheme 3).

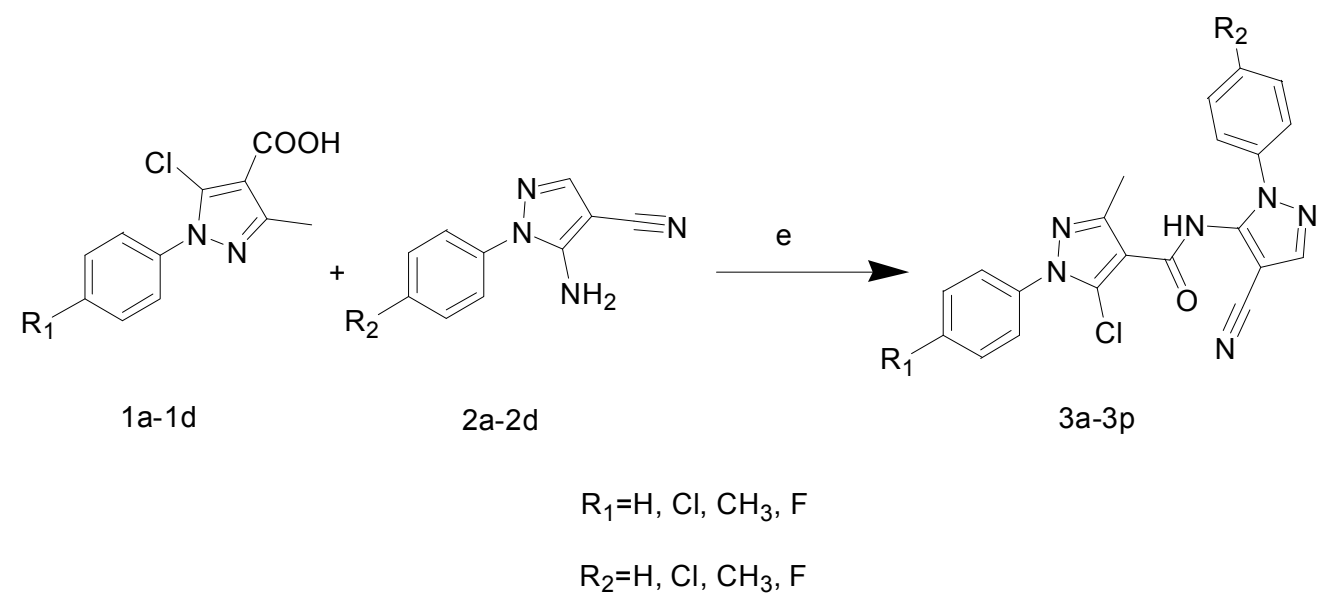

Scheme 3. General synthesis of compounds 3a-3p. (e) DMF, EDCI, HOBt, rt, TLC.

The structures of the newly synthesized compounds were confirmed by ${ }^{1} \mathrm{H}-\mathrm{NMR}$, mass spectroscopy and elemental analysis. In the ${ }^{1} \mathrm{H}-\mathrm{NMR}\left(\mathrm{CDCl}_{3}\right)$ spectra of the compounds, the N-H proton was hard to distinguish in the spectra. To prove its existence we used DMSO as solvent to dissolve compounds $\mathbf{3 e}$, 3f, 3n and then recorded the NMR spectra, whereupon the signal of compounds $\mathbf{3 e}$, $\mathbf{3 n}$ appears at 12.88 ppm and the signal of compounds $\mathbf{3} \mathbf{f}$ appears at $12.89 \mathrm{ppm}$. The NH group of compound $\mathbf{3 n}$ appears at $115.11 \mathrm{ppm}$ in the corresponding ${ }^{13} \mathrm{C}$-NMR spectrum taken in DMSO. The IR spectrum of compound 3b has been investigated in detail to exactly indicate the presence of $\mathrm{CN}$ groups and the main observed 
absorption bands were those located at: $3180.66 \mathrm{~cm}^{-1}(\mathrm{~N}-\mathrm{H}), 2230.44 \mathrm{~cm}^{-1}(\mathrm{C} \equiv \mathrm{N}), 1636.96 \mathrm{~cm}^{-1}(\mathrm{C}=\mathrm{O})$, $1560.03 \mathrm{~cm}^{-1}(\mathrm{C}=\mathrm{O}), 1404.77 \mathrm{~cm}^{-1}(\mathrm{C}-\mathrm{N}), 821.27 \mathrm{~cm}^{-1}$ (C-Cl arom), and $587.59 \mathrm{~cm}^{-1}$ (C-Cl pyrazole), which all support the proposed structure.

\subsection{Bioactivity}

To judgme the antiviral potency of the synthesized compounds $\mathbf{3 a}-\mathbf{3 p}$, the commercially available plant virucide ningnanmycin was used as the control. The antiviral bioassay against TMV was assayed by the method reported by Thorson et al. [37].

The in vivo and in vitro bioassay results of compounds $\mathbf{3 a - 3 p}$ against TMV are given in Table 1. The results indicated that the title compounds showed curative rates ranging from $22.6 \%-86.5 \%$. Among them, compound 3p showed the most potent biological activity against TMV and it exhibited slightly higher activities compared to the commercial agent ningnanmycin.

Table 1. Anti-TMV activity of compounds in vitro and in vivo at $500 \mathrm{mg} / \mathrm{mL}$.

\begin{tabular}{|c|c|c|c|c|}
\hline \multirow[b]{2}{*}{ Compound NO. } & \multirow{2}{*}{$\begin{array}{c}\text { In Vitro Inhibition } \\
\text { Rate }(\%)\end{array}$} & \multicolumn{3}{|c|}{ In Vivo Inhibition Rate (\%) } \\
\hline & & $\begin{array}{l}\text { Protection } \\
\text { Effect }\end{array}$ & $\begin{array}{c}\text { Inactivation } \\
\text { Effect }\end{array}$ & Curative Effect \\
\hline $3 a$ & $54.8 \pm 1.11$ & $32.4 \pm 1.45$ & $43.2 \pm 4.01$ & $47.3 \pm 0.88$ \\
\hline $\mathbf{3 b}$ & $66.4 \pm 0.78$ & $48.4 \pm 0.23$ & $54.2 \pm 2.11$ & $54.5 \pm 1.03$ \\
\hline $3 c$ & $52.1 \pm 1.20$ & $43.1 \pm 2.33$ & $42.6 \pm 1.09$ & $42.2 \pm 2.12$ \\
\hline 3d & $68.1 \pm 2.33$ & $60.2 \pm 2.11$ & $53.1 \pm 2.09$ & $55.1 \pm 2.15$ \\
\hline $3 e$ & $67.2 \pm 0.48$ & $49.3 \pm 1.03$ & $58.4 \pm 0.97$ & $56.5 \pm 0.55$ \\
\hline $3 f$ & $83.2 \pm 1.16$ & $76.7 \pm 2.54$ & $74.2 \pm 1.58$ & $78.5 \pm 0.82$ \\
\hline $3 g$ & $64.3 \pm 0.66$ & $33.8 \pm 3.56$ & $52.3 \pm 3.12$ & $41.2 \pm 3.20$ \\
\hline $3 \mathbf{h}$ & $81.6 \pm 0.72$ & $78.9 \pm 1.21$ & $66.4 \pm 4.23$ & $75.3 \pm 1.31$ \\
\hline $3 \mathbf{i}$ & $53.3 \pm 0.51$ & $22.6 \pm 1.36$ & $43.3 \pm 1.24$ & $52.1 \pm 2.11$ \\
\hline $3 \mathbf{j}$ & $42.3 \pm 0.48$ & $45.2 \pm 1.97$ & $52.6 \pm 0.65$ & $44.2 \pm 5.12$ \\
\hline $3 \mathbf{k}$ & $50.2 \pm 1.33$ & $42.1 \pm 1.29$ & $22.8 \pm 6.03$ & $38.9 \pm 4.33$ \\
\hline 31 & $64.6 \pm 4.11$ & $41.2 \pm 7.03$ & $45.3 \pm 7.34$ & $42.1 \pm 1.43$ \\
\hline $3 m$ & $74.4 \pm 2.08$ & $52.1 \pm 3.02$ & $64.6 \pm 0.63$ & $45.9 \pm 3.32$ \\
\hline $3 n$ & $84.3 \pm 1.52$ & $77.5 \pm 3.51$ & $74.5 \pm 2.13$ & $78.9 \pm 1.65$ \\
\hline 30 & $72.1 \pm 1.18$ & $61.3 \pm 1.30$ & $67.8 \pm 0.92$ & $52.1 \pm 2.06$ \\
\hline $3 p$ & $86.5 \pm 4.96$ & $77.2 \pm 6.03$ & $84.3 \pm 0.52$ & $81.5 \pm 1.03$ \\
\hline Ningnanmycin & $82.1 \pm 3.31$ & $62.4 \pm 7.20$ & $78.4 \pm 5.14$ & $55.2 \pm 6.12$ \\
\hline
\end{tabular}

\subsection{Molecular Docking}

Molecular docking of compound $\mathbf{4 p}$ into the three dimensional X-ray structure of the TMV PC protein-ligand complex crystal structure (PDB code: 2OM3) was carried out using the Discovery Studio (DS) (version 3.1, NeoTrident Corporation, Beijing, China) software [38] as implemented through the graphical user interface DS-CDOCKER protocol. The three-dimensional structures of the aforementioned compounds were constructed using Chem. 3D ultra 12.0 software (Chemical Structure Drawing Standard, CambridgeSoft Corporation, Cambridge, MA, USA), then they were energetically minimized by using MMFF94 with 5000 iterations and minimum RMS gradient of 0.10 . The crystal structures of protein complex were retrieved from the RCSB Protein Data Bank 
(http://www.rcsb.org/pdb/home/home.do) and prepared by Discovery Studio 3.1 with all bound waters and ligands eliminated from the protein and the polar hydrogen added to the protein. The molecular docking procedure was performed by using CDOCKER protocol for receptor-ligand interactions section of DS 3.1.

\section{4. $3 D-Q S A R$}

In order to further explain of structure and activity relationships of these compounds, the in vitro antiviral activity data were used to build the 3D-QSAR models, and the 3D-QSAR study was performed by means of the DS 3.5 software. The correlation coefficient $\mathrm{r}^{2}$ between observed and predicted activity of training set was found to be 0.899 , which meant the dependability of linear fit. The molecules aligned with the iso-surfaces of the 3D-QSAR model coefficients on electrostatic potential grids (Figure 5a) and van der Waals grids (Figure 5b) are listed.

$\mathbf{a}$

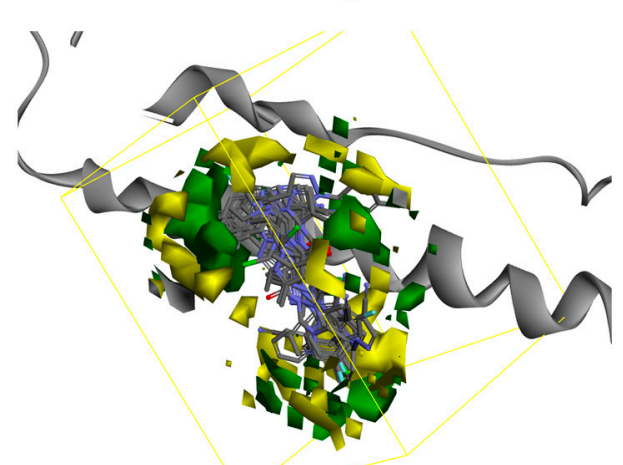

b

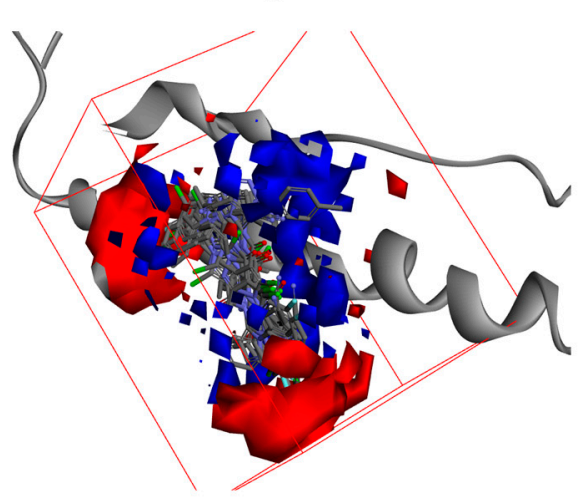

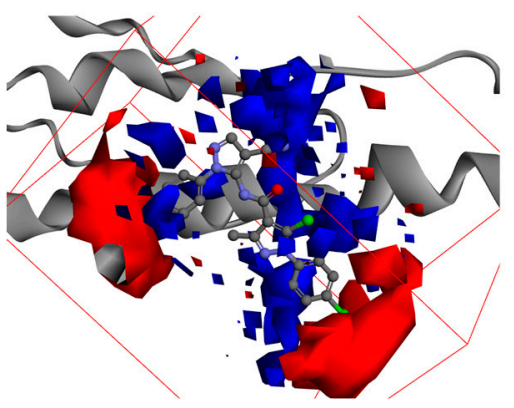

$3 \mathbf{f}$

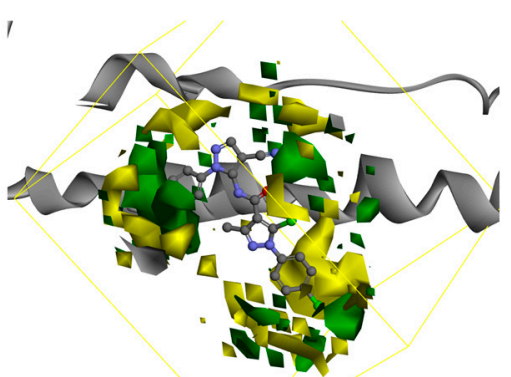

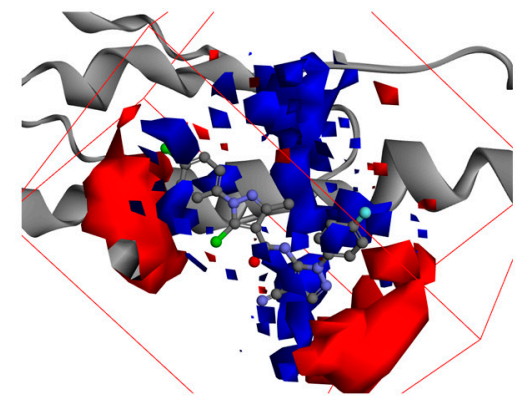

$3 \mathbf{h}$

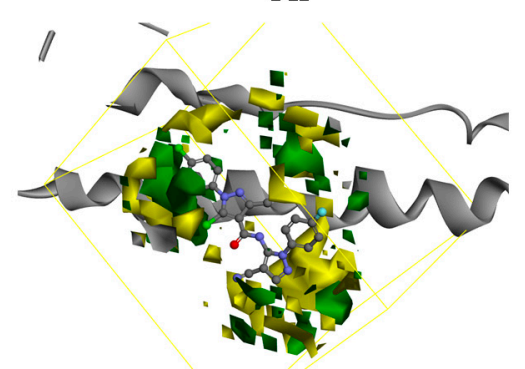

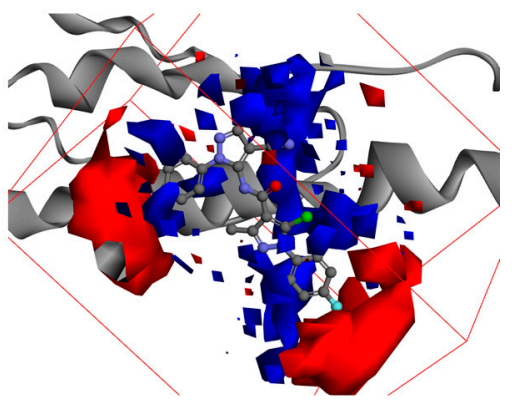

$3 \mathbf{p}$

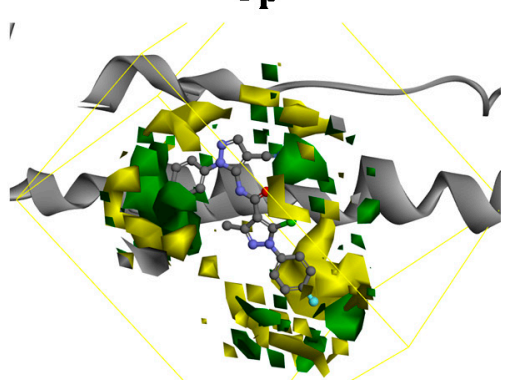

Figure 5. 3D-QSAR of pyrazole amide derivatives for TMV PC (PDB: 2OM3). (a) 3D-QSAR model coefficients on van der Waals grids. Green represents positive coefficients; yellow represents negative coefficients; (b) 3D-QSAR model coefficients on electrostatic potential grids. Blue represents positive coefficients; red represents negative coefficients. 
As shown in Figure 5, compounds with electron-withdrawing atoms not only circumvented the red subregion but also got more close to the favorable green spaces. This figure showed that a halogen substituted group was a better choice than a methyl substituted group and F was a better substituent than $\mathrm{Cl}$, so this QSAR study could predict that electron-withdrawing groupa substituted in the aromatic ring could have good interaction with the protein with nice molecule electrostatic and steric features. However, the chlorine atom is also a strong electron withdrawing-group but its ability is slight weaker than that of a $\mathrm{F}$ atom, therefore, the compounds with $\mathrm{Cl}$ atom substituted in the aromatic ring (e.g., 3f, 3h) have similar inhibition as 3p. The $3 \mathrm{D}$ QSAR models fitted the inhibitory activity well and thus provide us the directions for further modification.

\section{Experimental Section}

\subsection{General Information}

Melting points (uncorrected) were determined on an XT4 MP apparatus (Taike Corp., Beijing, China). ESI mass spectra were obtained on a Mariner System 5304 mass spectrometer (Applied Biosystems, Foster City, CA, USA). ${ }^{1} \mathrm{H}-\mathrm{NMR}$ spectra were recorded on a Bruker PX400 spectrometer (Bruker Corporation, Rheinstetten, Germany) at $25{ }^{\circ} \mathrm{C}$ with TMS and solvent signals assigned as internal references, ${ }^{13} \mathrm{C}$-NMR spectra and ${ }^{1} \mathrm{H}-\mathrm{NMR}$ spectra of compound 3e, $\mathbf{3 n}$ were recorded on an Agilent DD2 PX600 spectrometer (Agilent Technologies Corporation, Santa Clara, CA, USA). Chemical shifts were reported in ppm $(\delta)$. Elemental analyses were performed on a CHN-O-Rapid instrument (Leco, Tres Cantos, Madrid, Spain) and were within $0.4 \%$ of the theoretical values.

\subsection{General Procedure for Synthesis of 5-Chloro-1-aryl-3-methyl-1H-pyrazole-4-carboxylic Acids 1a-1d}

The intermediate 5-chloro-1-aryl-3-methyl-1H-pyrazole-4-carboxylic acids $\mathbf{1 a - 1 d}$ were synthesized as the following: para-substituted phenylhydrazines $(0.025 \mathrm{~mol})$ were reacted with ethyl acetoacetate $(0.025 \mathrm{~mol})$ in anhydrous ethanol to form a solid, which was then dissolved in a cold mixed solution of DMF $(20 \mathrm{~mL})$ and $\mathrm{POCl}_{3}(16 \mathrm{~mL})$, and stirred at $50-60{ }^{\circ} \mathrm{C}$. The resulting mixture was poured into ice-cold water, a saturated solution of sodium hydroxide was added to neutralize the mixture, and the solid precipitate was filtered, and washed with water. Then above product was oxidized by $\mathrm{KMnO}_{4}$ solution, stirred at $70-80{ }^{\circ} \mathrm{C}$. After cooling to room temperature the $\mathrm{pH}$ of the reaction mixture was adjusted to $\mathrm{pH} 7-8$ by the dropwise addition of $\mathrm{KOH}$ solution, and the solution was filtered, $\mathrm{HCl}$ solution was added to the solution and solid 1a-1d eventually separated out. The crude product obtained was recrystallized from DMF to afford the pure product. All the reactions were monitored by TLC.

\subsection{General Procedure for Synthesis of 5-Amino-1-aryl-1H-pyrazole-4-carbonitriles 2a-2d}

A stirred mixture of para-substituted phenylhydrazine hydrochloride $(0.025 \mathrm{~mol})$ was dissolved in $\mathrm{H}_{2} \mathrm{O}(30 \mathrm{~mL})$, then the $\mathrm{pH}$ of the mixture was adjusted to $\mathrm{pH} 7-8$ by the dropwise addition of $10 \% \mathrm{NaOH}$ solution to form the free para-substituted phenyl hydrazines, which were then refluxed for $3 \mathrm{~h}$ with ethoxymethylene malononitrile in an ethanol medium. After completion of the reaction, the reaction mixture was allowed to cool at room temperature, and the solid $\mathbf{2 a - 2 d}$ were filtered under vacuum. The crude products obtained were recrystallized from DMF to afford the pure products. 
3.4. General Procedure for Synthesis of 5-Chloro-N-(4-cyano-1-aryl-1H-pyrazol-5-yl)-1-aryl-3methyl-1H-pyrazole-4-carboxamide Derivatives 3a-3p

To a stirred solution of the intermediate compounds $1 \mathbf{a}-\mathbf{1 d}(1 \mathrm{mmol})$ and triethylamine $(2 \mathrm{mmol})$ in DMF $(12 \mathrm{~mL})$ medium, a mixture of EDCI $(1 \mathrm{mmol})$ and HOBt $(1 \mathrm{mmol})$ was added and the reaction mixture was stirred at room temperature for $30 \mathrm{~min}$, then a mixture of compounds $\mathbf{2 a}-\mathbf{2 d}(1 \mathrm{mmol})$ and DMF ( $5 \mathrm{~mL}$ ) was added, the reaction was stirred at room temperature. And the reaction progress was monitored by TLC. After completion of the reaction, the product was added into chloroform, then extracted from chloroform with water, and washed successively with $0.2 \mathrm{~mol} / \mathrm{L}$ hydrochloric acid, water, $2 \mathrm{~mol} / \mathrm{L}$ sodium hydroxide, water, saturated sodium chloride, then dried, concentrated and purified by preparative thin layer chromatography $(\mathrm{PE}: \mathrm{EA}=8: 1)$ followed by recrystallization from ethanol.

5-Chloro-N-(4-cyano-1-phenyl-1H-pyrazol-5-yl)-3-methyl-1-phenyl-1H-pyrazole-4-carboxamide (3a). White crystals, yield 68\%, mp: $133-135^{\circ} \mathrm{C} .{ }^{1} \mathrm{H}-\mathrm{NMR}\left(400 \mathrm{MHz}, \mathrm{CDCl}_{3}\right) \delta: 8.12(\mathrm{~s}, 1 \mathrm{H}), 7.61-7.43$ (m, $10 \mathrm{H}), 2.66$ (s, 3H). MS (ESI): $403.1\left(\mathrm{C}_{21} \mathrm{H}_{15} \mathrm{ClN}_{6} \mathrm{O},[\mathrm{M}+\mathrm{H}]^{+}\right)$. Anal. Calcd for $\mathrm{C}_{21} \mathrm{H}_{15} \mathrm{ClN}_{6} \mathrm{O}: \mathrm{C}, 62.61$; H, 3.75; N, 20.86; Found: C, 62.82; H, 3.84; N, 21.04.

5-Chloro-1-(4-chlorophenyl)-N-(4-cyano-1-phenyl-1H-pyrazol-5-yl)-3-methyl-1H-pyrazole-4-carboxamide (3b). White crystals, yield 68\%. mp: $171-172{ }^{\circ} \mathrm{C}$, IR $\left(\mathrm{cm}^{-1}\right): 3180.66,2230.44,1636.96,1560.03$, 1404.77, 821.27, 587.59. ${ }^{1} \mathrm{H}-\mathrm{NMR}\left(400 \mathrm{MHz}, \mathrm{CDCl}_{3}\right) \delta: 8.12(\mathrm{~s}, 1 \mathrm{H}), 7.55-7.43$ (m, 9H), 2.65 (s, 3H). MS (ESI): $437.1\left(\mathrm{C}_{21} \mathrm{H}_{14} \mathrm{Cl}_{2} \mathrm{~N}_{6} \mathrm{O},[\mathrm{M}+\mathrm{H}]^{+}\right)$. Anal. Calcd for $\mathrm{C}_{21} \mathrm{H}_{14} \mathrm{Cl}_{2} \mathrm{~N}_{6} \mathrm{O}$ : C, 57.68; H, 3.23; N, 19.22; Found: C, 57.72; H, 3.31; N, 19.11.

5-Chloro-N-(4-cyano-1-(p-tolyl)-1H-pyrazol-5-yl)-3-methyl-1-phenyl-1H-pyrazole-4-carboxamide (3c). White crystals, yield 62\%, mp: $123-125{ }^{\circ} \mathrm{C} ;{ }^{1} \mathrm{H}-\mathrm{NMR}\left(400 \mathrm{MHz}, \mathrm{CDCl}_{3}\right) \delta: 8.12$ (s, 1H), 7.63-7.41 (m, 9H), $2.66(\mathrm{~s}, 3 \mathrm{H}), 2.42(\mathrm{~s}, 3 \mathrm{H})$. MS (ESI): $417.1\left(\mathrm{C}_{22} \mathrm{H}_{17} \mathrm{ClN} 6 \mathrm{O},[\mathrm{M}+\mathrm{H}]^{+}\right)$. Anal. Calcd for $\mathrm{C}_{22} \mathrm{H}_{17} \mathrm{ClN}_{6} \mathrm{O}: \mathrm{C}$, 63.39; H, 4.11; N, 20.16; Found: C, 63.71; H, 4.42; N, 20.22.

5-Chloro-N-(4-cyano-1-(4-fluorophenyl)-1H-pyrazol-5-yl)-3-methyl-1-phenyl-1H-pyrazole-4-carboxamide (3d). White crystal, yield 71\%, mp: $181-183{ }^{\circ} \mathrm{C} .{ }^{1} \mathrm{H}-\mathrm{NMR}\left(400 \mathrm{MHz}, \mathrm{CDCl}_{3}\right) \delta: 8.12(\mathrm{~s}, 1 \mathrm{H})$, 7.51-7.21 (m, 9H), $2.66(\mathrm{~s}, 3 \mathrm{H})$. MS (ESI):421.0 $\left(\mathrm{C}_{21} \mathrm{H}_{14} \mathrm{ClFN}_{6} \mathrm{O},[\mathrm{M}+\mathrm{H}]^{+}\right)$. Anal. Calcd for $\mathrm{C}_{21} \mathrm{H}_{14} \mathrm{ClFN}_{6} \mathrm{O}$ : C, 59.54; H, 3.35; N, 19.97; Found: C, 59.72; H, 3.41; N, 20.06.

5-Chloro-1-(4-chlorophenyl)-N-(4-cyano-1-phenyl-1H-pyrazol-5-yl)-3-methyl-1H-pyrazole-4-carboxamide (3e). White crystals, yield 66\%, mp: $172-174{ }^{\circ} \mathrm{C} .{ }^{1} \mathrm{H}-\mathrm{NMR}\left(400 \mathrm{MHz}, \mathrm{CDCl}_{3}\right) \delta: 8.12(\mathrm{~s}, 1 \mathrm{H})$, 7.61-7.43 (m, 9H), 2.65 (s, 3H). ${ }^{1} \mathrm{H}-\mathrm{NMR}\left(600 \mathrm{MHz}, \mathrm{DMSO}-d_{6}\right) \delta: 12.88(\mathrm{~s}, 1 \mathrm{H}), 7.97(\mathrm{~s}, 1 \mathrm{H})$, 7.62-7.41 (m, 9H), 2.48 (s, 3H). MS (ESI): $437.1\left(\mathrm{C}_{21} \mathrm{H}_{14} \mathrm{Cl}_{2} \mathrm{~N}_{6} \mathrm{O},[\mathrm{M}+\mathrm{H}]^{+}\right)$. Anal. Calcd for $\mathrm{C}_{21} \mathrm{H}_{14} \mathrm{Cl}_{2} \mathrm{~N}_{6} \mathrm{O}$ : C, 57.68; H, 3.23; N, 19.22; Found: C, 57.74; H, 3.41; N, 19.42.

5-Chloro-1-(4-chlorophenyl)-N-(1-(4-chlorophenyl)-4-cyano-1H-pyrazol-5-yl)-3-methyl-1H-pyrazole4-carboxamide (3f). White crystals, yield 79\%, mp: 178-180 ${ }^{\circ} \mathrm{C} .{ }^{1} \mathrm{H}-\mathrm{NMR}\left(400 \mathrm{MHz}, \mathrm{DMSO}-d_{6}\right) \delta: 12.89$ (s, 1H), $7.98(\mathrm{~s}, 1 \mathrm{H}), 7.63-7.42(\mathrm{~m}, 8 \mathrm{H}), 2.65$ (s, 3H). MS (ESI): $471.0\left(\mathrm{C}_{21} \mathrm{H}_{13} \mathrm{Cl}_{3} \mathrm{~N}_{6} \mathrm{O},[\mathrm{M}+\mathrm{H}]^{+}\right)$. Anal. Calcd for $\mathrm{C}_{21} \mathrm{H}_{13} \mathrm{Cl}_{3} \mathrm{~N}_{6} \mathrm{O}$ : C, 53.47; H, 2.78; N, 17.82; Found: C, 53.56; H, 2.81; N, 17.91 . 
5-Chloro-1-(4-chlorophenyl)-N-(4-cyano-1-(p-tolyl)-1H-pyrazol-5-yl)-3-methyl-1H-pyrazole-4carboxamide (3g). White crystals, yield 69\%, mp: $130-132{ }^{\circ} \mathrm{C} .{ }^{1} \mathrm{H}-\mathrm{NMR}\left(400 \mathrm{MHz}, \mathrm{CDCl}_{3}\right) \delta: 8.12$ (s, $1 \mathrm{H}), 7.60-7.43(\mathrm{~m}, 8 \mathrm{H}), 2.65(\mathrm{~s}, 3 \mathrm{H}), 2.42(\mathrm{~s}, 3 \mathrm{H})$. MS (ESI): $451.1\left(\mathrm{C}_{22} \mathrm{H}_{16} \mathrm{Cl}_{2} \mathrm{~N}_{6} \mathrm{O},[\mathrm{M}+\mathrm{H}]^{+}\right)$. Anal. Calcd for $\mathrm{C}_{22} \mathrm{H}_{16} \mathrm{Cl}_{2} \mathrm{~N}_{6} \mathrm{O}$ : C, 58.55; H, 3.57; N, 18.62; Found: C, 58.58; H, 3.71; N, 18.74.

5-Chloro-1-(4-chlorophenyl)-N-(4-cyano-1-(4-fluorophenyl)-1H-pyrazol-5-yl)-3-methyl-1H-pyrazole4-carboxamide (3h). White crystals, yield 69\%, mp: 177-178 ${ }^{\circ} \mathrm{C} .{ }^{1} \mathrm{H}-\mathrm{NMR}\left(400 \mathrm{MHz}, \mathrm{CDCl}_{3}\right) \delta: 8.12$ (s, 1H), 7.53-7.20 (m, 8H), $2.66(\mathrm{~s}, 3 \mathrm{H})$. MS (ESI): $455.1\left(\mathrm{C}_{21} \mathrm{H}_{13} \mathrm{Cl}_{2} \mathrm{FN} 6 \mathrm{O},[\mathrm{M}+\mathrm{H}]^{+}\right)$. Anal. Calcd for $\mathrm{C}_{21} \mathrm{H}_{13} \mathrm{Cl}_{2} \mathrm{FN}_{6} \mathrm{O}$ : C, 55.40; H, 2.88; N, 18.46; Found: C, 55.51; H, 2.93; N, 18.48 .

5-Chloro-N-(4-cyano-1-phenyl-1H-pyrazol-5-yl)-3-methyl-1-(p-tolyl)-1H-pyrazole-4-carboxamide (3i). White crystals, yield 64\%, mp: $124-126{ }^{\circ} \mathrm{C} .{ }^{1} \mathrm{H}-\mathrm{NMR}\left(400 \mathrm{MHz}, \mathrm{CDCl}_{3}\right) \delta: 8.12(\mathrm{~s}, 1 \mathrm{H}), 7.62-7.41$ (m, 9H), $2.65(\mathrm{~s}, 3 \mathrm{H}), 2.42(\mathrm{~s}, 3 \mathrm{H})$. MS (ESI): $417.1\left(\mathrm{C}_{22} \mathrm{H}_{17} \mathrm{ClN}_{6} \mathrm{O},[\mathrm{M}+\mathrm{H}]^{+}\right)$. Anal. Calcd for $\mathrm{C}_{22} \mathrm{H}_{17} \mathrm{ClN}_{6} \mathrm{O}$ : C, 63.39; H, 4.11; N, 20.16; Found: C, 63.44; H, 4.14; N, 20.18.

5-Chloro-N-(1-(4-chlorophenyl)-4-cyano-1H-pyrazol-5-yl)-3-methyl-1-(p-tolyl)-1H-pyrazole-4carboxamide (3j). White crystals, yield 77\%, mp: $155-157{ }^{\circ} \mathrm{C} .{ }^{1} \mathrm{H}-\mathrm{NMR}\left(400 \mathrm{MHz}, \mathrm{CDCl}_{3}\right) \delta: 8.11$ (s, 1H), 7.61-7.41 (m, 8H), $2.65(\mathrm{~s}, 3 \mathrm{H}), 2.46$ (s, 3H). MS (ESI): $451.1\left(\mathrm{C}_{22} \mathrm{H}_{16} \mathrm{Cl}_{2} \mathrm{~N}_{6} \mathrm{O},[\mathrm{M}+\mathrm{H}]^{+}\right)$. Anal. Calcd for $\mathrm{C}_{22} \mathrm{H}_{16} \mathrm{Cl}_{2} \mathrm{~N}_{6} \mathrm{O}$ : C, 58.55; H, 3.57; N, 18.62; Found: C, 58.58; H, 3.61; N, 18.66.

5-Chloro-N-(4-cyano-1-(p-tolyl)-1H-pyrazol-5-yl)-3-methyl-1-(p-tolyl)-1H-pyrazole-4-carboxamide (3k). White crystals, yield 63\%, mp: $133-135^{\circ} \mathrm{C} .{ }^{1} \mathrm{H}-\mathrm{NMR}\left(400 \mathrm{MHz}, \mathrm{CDCl}_{3}\right) \delta: 8.12(\mathrm{~s}, 1 \mathrm{H}), 7.61-7.43$ (m, $8 \mathrm{H}), 2.65(\mathrm{~s}, 3 \mathrm{H}), 2.42(\mathrm{~s}, 6 \mathrm{H})$. MS (ESI): $431.1\left(\mathrm{C}_{23} \mathrm{H}_{19} \mathrm{ClN}_{6} \mathrm{O},[\mathrm{M}+\mathrm{H}]^{+}\right)$. Anal. Calcd for $\mathrm{C}_{23} \mathrm{H}_{19} \mathrm{ClN}_{6} \mathrm{O}$ : C, 64.11; H, 4.44; N, 19.50; Found: C, 64.17; H, 4.47; N, 19.56.

5-Chloro-N-(4-cyano-1-(4-fluorophenyl)-1H-pyrazol-5-yl)-3-methyl-1-(p-tolyl)-1H-pyrazole-4-carboxamide (3l). White crystals, yield 62\%, mp: $156-158{ }^{\circ} \mathrm{C} .{ }^{1} \mathrm{H}-\mathrm{NMR}\left(400 \mathrm{MHz}, \mathrm{CDCl}_{3}\right) \delta: 8.12(\mathrm{~s}, 1 \mathrm{H})$, 7.61-7.22 (m, 8H), $2.65(\mathrm{~s}, 3 \mathrm{H}), 2.42(\mathrm{~s}, 3 \mathrm{H}) . \mathrm{MS}(\mathrm{ESI}): 435.1\left(\mathrm{C}_{22} \mathrm{H}_{16} \mathrm{ClFN}_{6} \mathrm{O},[\mathrm{M}+\mathrm{H}]^{+}\right)$. Anal. Calcd for $\mathrm{C}_{22} \mathrm{H}_{16} \mathrm{ClFN}_{6} \mathrm{O}$ : C, 60.76; H, 3.71; N, 19.33; Found: C, 60.77; H, 3.74; N, 19.46.

5-Chloro-N-(4-cyano-1-phenyl-1H-pyrazol-5-yl)-1-(4-fluorophenyl)-3-methyl-1H-pyrazole-4-carboxamide (3m). White crystals, yield 69\%, mp: $118-120{ }^{\circ} \mathrm{C} .{ }^{1} \mathrm{H}-\mathrm{NMR}\left(400 \mathrm{MHz}, \mathrm{CDCl}_{3}\right) \delta: 8.12(\mathrm{~s}, 1 \mathrm{H})$, 7.61-7.23 (m, 9H), 2.65 (s, 3H). MS (ESI): $421.1\left(\mathrm{C}_{21} \mathrm{H}_{14} \mathrm{ClFN}_{6} \mathrm{O},[\mathrm{M}+\mathrm{H}]^{+}\right)$. Anal. Calcd for $\mathrm{C}_{22} \mathrm{H}_{16} \mathrm{ClN}_{6} \mathrm{O}$ : C, 59.94; H, 3.35; N, 19.97; Found: C, 60.07; H, 3.43; N, 20.04.

5-Chloro-N-(1-(4-chlorophenyl)-4-cyano-1H-pyrazol-5-yl)-1-(4-fluorophenyl)-3-methyl-1H-pyrazole4-carboxamide (3n). White crystals, yield 81\%, mp: $149-151{ }^{\circ} \mathrm{C} .{ }^{1} \mathrm{H}-\mathrm{NMR}\left(400 \mathrm{MHz}, \mathrm{CDCl}_{3}\right) \delta: 8.12$ $(\mathrm{s}, 1 \mathrm{H}), 7.62-7.22(\mathrm{~m}, 8 \mathrm{H}), 2.65(\mathrm{~s}, 3 \mathrm{H}) .{ }^{1} \mathrm{H}-\mathrm{NMR}\left(600 \mathrm{MHz}, \mathrm{DMSO}-d_{6}\right) \delta: 12.88(\mathrm{~s}, 1 \mathrm{H}), 7.97(\mathrm{~s}, 1 \mathrm{H})$, 7.62-7.21 (m, 8H), 2.48 (s, 3H). ${ }^{13} \mathrm{C}-\mathrm{NMR}\left(151 \mathrm{MHz}, \mathrm{DMSO}-d_{6}\right) \delta 163.47,163.14,151.88,151.73$, $142.43,136.76,133.97,132.68,131.25,128.64,128.58,116.71,116.56,115.11,110.46,73.96,14.86$. MS (ESI): $455.1\left(\mathrm{C}_{21} \mathrm{H}_{13} \mathrm{Cl}_{2} \mathrm{FN}_{6} \mathrm{O},[\mathrm{M}+\mathrm{H}]^{+}\right)$. Anal. Calcd for $\mathrm{C}_{21} \mathrm{H}_{13} \mathrm{Cl}_{2} \mathrm{FN}_{6} \mathrm{O}: \mathrm{C}, 55.40 ; \mathrm{H}, 2.88 ; \mathrm{N}$, 18.46; Found: C, 55.53; H, 2.91; N, 18.49 . 
5-Chloro-N-(4-cyano-1-(p-tolyl)-1H-pyrazol-5-yl)-1-(4-fluorophenyl)-3-methyl-1H-pyrazole-4-carboxamide (3o). White crystals, yield 65\%, mp: $127-129{ }^{\circ} \mathrm{C} .{ }^{1} \mathrm{H}-\mathrm{NMR}\left(400 \mathrm{MHz}, \mathrm{CDCl}_{3}\right) \delta: 8.12(\mathrm{~s}, 1 \mathrm{H})$, 7.61-7.22 (m, 8H), 2.65 (s, 3H), 2.42 (s, 3H). MS (ESI): $435.1\left(\mathrm{C}_{22} \mathrm{H}_{16} \mathrm{ClFN}_{6} \mathrm{O},[\mathrm{M}+\mathrm{H}]^{+}\right)$. Anal. Calcd for $\mathrm{C}_{22} \mathrm{H}_{16} \mathrm{ClFN}_{6} \mathrm{O}$ : C, 60.76; H, 3.71; N, 19.33; Found: C, 60.80; H, 3.77; N, 19.49.

5-Chloro-N-(4-cyano-1-(4-fluorophenyl)-1H-pyrazol-5-yl)-1-(4-fluorophenyl)-3-methyl-1H-pyrazole4-carboxamide (3p). White crystals, yield 62\%, mp: $182-183{ }^{\circ} \mathrm{C} .{ }^{1} \mathrm{H}-\mathrm{NMR}\left(400 \mathrm{MHz}, \mathrm{CDCl}_{3}\right) \delta: 8.12$ (s, 1H), 7.61-7.21 (m, 8H), 2.65 (s, 3H). MS (ESI): $439.1\left(\mathrm{C}_{21} \mathrm{H}_{13} \mathrm{ClF}_{2} \mathrm{~N}_{6} \mathrm{O},[\mathrm{M}+\mathrm{H}]^{+}\right)$. Anal. Calcd for $\mathrm{C}_{21} \mathrm{H}_{13} \mathrm{ClF}_{2} \mathrm{~N}_{6} \mathrm{O}$ : C, 57.48; H, 2.99; N, 19.15; Found: C, 57.53; H, 3.03; N, 19.17.

\subsection{Antiviral Biological Assay}

The anti-TMV activity of the synthesized pure compounds were tested by using the method reported by Thorson et al. [37]. This method is to measure the antiviral activity of compounds in vitro and to test the protective effect, the inactivation effect and the curative effect in vivo against TMV.

\subsubsection{Antiviral Activity of Target Compounds in Vitro}

Fresh 5-6 growth stage of tobacco leaves (Nicotianatabacum var. XanthiNC) were selected for the test. The tobacco was inoculated by the juice-leaf rubbing method, and the concentration of TMV was $5.88 \times 10^{-2} \mu \mathrm{g} / \mathrm{mL}$. The leaves was cut into halves along the main vein, and a solution of the compounds with a concentration of $500 \mu \mathrm{g} / \mathrm{mL}$ was smeared on the halves, and then cultured at $25^{\circ} \mathrm{C}$ for $72 \mathrm{~h}$. Each compound was tested three times.

\subsubsection{Protective Effect of Target Compounds against TMV in Vivo}

The test compound solution was smeared on the left side, and the solvent served as control on the right side of growing tobacco (Nicotianatabacum var. XanthiNC). After $12 \mathrm{~h}$, TMV at a concentration of $6.0 \mu \mathrm{g} / \mathrm{mL}$ was inoculated on the leaves which were previously scattered with silicon carbide through the above juice-leaf rubbing method. Then the leaves were again immersed into water and rubbed softly along the nervation once or twice. The local lesion numbers showing 3-4 days after inoculation were counted. Each compound was tested in three replicates.

\subsubsection{Inactivate Effect of Target Compounds against TMV in Vivo}

The virus was inhibited by mixing with the target compound solution at the same volume for $30 \mathrm{~min}$. Then the mixture was inoculated on the left side of the host tobacco leaves (Nicotianatabacum var. XanthiNC), and a mixture of solvent and the virus was inoculated on the right side to serve as control. The local lesion numbers showing 3-4 days after inoculation were counted. Each compound was tested in three replicates.

\subsubsection{Curative Effect of Target Compounds against TMV in Vivo}

Host plant tobacco leaves (Nicotianatabacum var. XanthiNC) of the same age growing at the six-leaf stage were selected for the test. TMV at a concentration of $6.0 \mu \mathrm{g} / \mathrm{mL}$ was inoculated on the whole 
leaves. The leaves were washed with water, and dried in a greenhouse. The target compound solution was smeared on the left side, and the solvent served as control on the right side. The local lesion numbers showing 3-4 days after inoculation were counted. Each compound was tested in three replicates. The inhibition rates of the compound in vitro and in vivo were calculated according to the following formula (controls were not treated with compound):

Inhibition rate $(\%)=[($ average local lesion number of control - average local lesion number of drug treated)/average local lesion number of control] $\times 100 \%$

\section{Conclusions}

In conclusion, a series of novel pyrazole amide compounds has been designed and synthesized, based on the skeleton of previously reported pyrazole derivatives. The title compounds $\mathbf{3 a}-\mathbf{3 p}$ were evaluated as potent antiviral agents against TMV and it was proved that compound $\mathbf{3 p}$ showed a significant activity.

Structure-activity relationships in these pyrazole amide derivatives were evaluated using the DS approach to proceed with structure optimization, The data demonstrated that compounds with para electron-withdrawing substituents (e.g., 3f, 3h, 3n, 3p) in the A-ring showed more potent activities than those with electron-donating substituents (e.g., 3a, 3d, 3i, 3k). In comparison the activity of compound with para substituents on the phenyl ring showed that an electron-withdrawing group gave slightly improved antiviral activity and the potency order is $\mathrm{F}>\mathrm{Cl}$. Among all the compounds, 3p with a para-F group showed more activity, while $3 \mathbf{k}$ showed the lowest activity.

For the binding model of compound 3p, an H-bond $\left(2.49 \AA\right.$ and $\left.109.02^{\circ}\right)$ was established between its fluorine atom and Gin47, and it also formed a $\pi$-cation interaction (4.65 $\AA$ ). The result of molecular docking suggests that introduction of a fluorine atom facilitates the adoption of a complementary shape of the designed ligands with TMV PC, and this possibly improves the anti-TMV inhibitory potency.

\section{Acknowledgments}

This work was supported by National Natural Science Foundation of China (No. 21302002) and Anhui Natural Science Foundation (1408085QB33).

\section{Author Contributions}

Hai-Qun Cao, Xian-Hai Lv, and Jin-Jing Xiao conceived and designed the study. Jin-Jing Xiao, and Zi-Li Ren performed the experiments. Ming-Jie Chu and Xin Zhang analyzed data. Jin-Jing Xiao wrote the manuscript. Min Liao edited and revised the manuscript.

\section{Conflicts of Interest}

The authors declare no conflict of interest.

\section{References}

1. Ren, Y.P.; Wong, S.M.; Lim, L.Y. Application of plant viruses as nano drug delivery systems. Pharm. Res. 2010, 27, 2509-2513. 
2. Ritzenthaler, C. Resistance to plant viruses: Old issue, news answers? Curr. Opin. Biotechnol. 2005, 16, 118-122.

3. Wang, Z.; Wei, P.; Wang, L.; Wang, Q. Design, synthesis, and anti-tobacco mosaic virus (TMV) activity of phenanthroindolizidines and their analogues. J. Agric. Food Chem. 2012, 60, 10212-10219.

4. Wang, Z.; Wei, P.; Xizhi, X.; Liu, Y.; Wang, L.; Wang, Q. Design, synthesis, and antiviral activity evaluation of phenanthrene-based antofine derivatives. J. Agric. Food Chem. 2012, 60, 8544-8551.

5. Whiting, M.; Muldoon, J.; Lin, Y.C.; Silverman, S.M.; Lindstrom, W.; Olson, A.J.; Kolb, H.C.; Finn, M.G.; Sharpless, K.B.; Elder, J.H.; et al. Inhibitors of HIV-1 protease by using in situ click chemistry. Angew. Chem. Int. Ed. 2006, 45, 1435-1439.

6. Ghosh, A.K.; Chapsal, B.D.; Weber, I.T.; Mitsuya, H. Design of HIV protease inhibitors targeting protein backbone: An effective strategy for combating drug resistance. Acc. Chem. Res. 2008, 41, 78-86.

7. Bendahmane, M.; Chen, I.; Asurmendi, S.; Bazzini, A.A.; Szecsi, J.; Beachy, R.N. Coat protein-mediated resistance to TMV infection of Nicotiana tabacum involves multiple modes of interference by coat protein. Virology 2007, 366, 107-116.

8. Matthews, R. Plant Virology; Elsevier: Amsterdam, The Netherlands, 1991; p. 864.

9. Ko, H.C.; Wei, B.L.; Chiou, W.F. The effect of medicinal plants used in Chinese folk medicine on RANTES secretion by virus-infected human epithelial cells. J. Ethnopharmacol. 2006, 107, 205-210.

10. Song, B.; Jin, L.; Yang, S.; Bhadury, P.S. Environment-Friendly Antiviral Agents for Plants, 1st ed.; Chemical Industry Press: Beijing, China, 2010.

11. Gao, S.; Zhang, R.Y.; Yu, Z.H.; Xi, Z. Antofine analogues can inhibit tobacco mosaic virus assembly through small-molecule-RNA interactions. Chembiochem 2012, 13, 1622-1627.

12. Thomas, J.R.; Hergenrother, P.J. Targeting RNA with small molecules. Chem. Rev. 2008, 108, 1171-1224.

13. Graillot, V.; Tomasetig, F.; Cravedi, J.-P.; Audebert, M. Evidence of the in vitro genotoxicity of methyl-pyrazole pesticides in human cells. Mutat. Res. 2012, 748, 8-16.

14. Giornal, F.; Pazenok, S.; Rodefeld, L.; Lui, N.; Vors, J.-P.; Leroux, F.R. Synthesis of diversely fluorinated pyrazoles as novel active agrochemical ingredients. J. Fluorine Chem. 2013, 152, 2-11.

15. Xue, N.; Zhou, Y.; Wang, G.; Miao, W.; Qu, J. Syntheses and herbicidal activity of pyrazolyl benzoxazole derivatives. ChemInform 2010, 41, 15-21.

16. Mali, J.R.; Pratap, U.R.; Jawale, D.V.; Mane, R.A. Water-mediated one-pot synthetic route for pyrazolo[3,4-b]quinolines. Tetrahedron Lett. 2010, 51, 3980-3982.

17. Al-Masoudi, I.A.; Al-Souda, Y.A.; Al-Salihi, N.J.; Al-Masoudi, N.A. 1,2,4-Triazoles: Synthetic approaches and pharmacological importance. Chem. Heterocycl. Comp. 2006, 42, 1377-1403.

18. Kumari, S.; Paliwal, S.; Chauhan, R. Synthesis of pyrazole derivatives possessing anticancer activity: Current status. Synth. Commun. 2014, 44, 1521-1578.

19. Rida, S.M.; Ashour, F.A.; El-Hawash, S.A.M.; Elsemary, M.M.; Badr, M.H.; Shalaby, M.A. Synthesis of some novel benzoxazole derivatives as anticancer, anti-HIV-1 and antimicrobial agents. Eur. J. Med. Chem. 2005, 40, 949-959. 
20. Siddall, T.L.; Ouse, D.G.; Benko, Z.L.; Garvin, G.M.; Jackson, J.L.; McQuiston, J.M.; Ricks, M.J.; Thibault, T.D.; Turner, J.A.; Vanheertum, J.C.; et al. Synthesis and herbicidal activity of phenyl-substituted benzoylpyrazoles. Pest Manag. Sci. 2002, 58, 1175-86.

21. Meazza, G.; Bettarini, F.; La Porta, P.; Piccardi, P.; Signorini, E.; Portoso, D.; Fornara, L. Synthesis and herbicidal activity of novel heterocyclic protoporphyrinogen oxidase inhibitors. Pest Manag. Sci. 2004, 60, 1178-1188.

22. Liu, M.Y.; Shi, D.Q. Synthesis and herbicidal activity of 2-aroxy-propanamides containing pyrimidine and 1,3,4-thiadiazole rings. J. Heterocycl. Chem. 2014, 51, 432-435.

23. Satasia, S.P.; Kalaria, P.N.; Raval, D.K. Catalytic regioselective synthesis of pyrazole based pyrido[2,3-d]pyrimidine-diones and their biological evaluation. Org. Biomol. Chem. 2014, 12, 1751-1758.

24. Rahmouni, A.; Romdhane, A.; Ben Said, A.; Majouli, K.; Ben Jannet, H. Synthesis of new pyrazole and antibacterial pyrazolopyrimidine derivatives. Turk. J. Chem. 2014, 38, 210-221.

25. Darwish, E.S.; Fattah, A.M.A.; Attaby, F.A.; Al-Shayea, O.N. Synthesis and antimicrobial evaluation of some novel thiazole, pyridone, pyrazole, chromene, hydrazone derivatives bearing a biologically active sulfonamide moiety. Int. J. Mol. Sci. 2014, 15, 1237-1254.

26. Lamberth, C. Pyrazole chemistry in crop protection. Heterocycles 2007, 71, 1467-1502.

27. Jiang, D.X.; Zheng, X.H.; Shao, G.; Ling, Z.; Xu, H.H. Discovery of a novel series of phenyl pyrazole inner salts based on fipronil as potential dual-target insecticides. J. Agric. Food Chem. 2014, 62, 3577-3583.

28. Ouyang, G.P.; Cai, X.J.; Chen, Z.; Song, B.A.; Bhadury, P.S.; Yang, S.; Jin, L.H.; Xue, W.; Hu, D.Y.; Zeng, S. Synthesis and antiviral activities of pyrazole derivatives containing an oxime moiety. J. Agric. Food Chem. 2008, 56, 10160-10167.

29. Li, Y.; Zhang, H.Q.; Liu, J.; Yang, X.P.; Liu, Z.J. Stereoselective synthesis and antifungal activities of $(E)$-alpha-(methoxyimino)benzeneacetate derivatives containing 1,3,5-substituted pyrazole ring. J. Agric. Food Chem. 2006, 54, 3636-3640.

30. Ouyang, G.P.; Chen, Z.; Cai, X.J.; Song, B.A.; Bhadury, P.S.; Yang, S.; Jin, L.H.; Xue, W.; Hu, D.Y.; Zeng, S. Synthesis and antiviral activity of novel pyrazole derivatives containing oxime esters group. Bioorganic Med. Chem. 2008, 16, 9699-9707.

31. Reid, T.S.; Beese, L.S. Crystal structures of the anticancer clinical candidates R1 15777 (Tipifarnib) and BMS-214662 complexed with protein farnesyltransferase suggest a mechanism of FTI selectivity. Biochemistry 2004, 43, 6877-6884.

32. Hunt, J.T.; Ding, C.Z.; Batorsky, R.; Bednarz, M.; Bhide, R.; Cho, Y.; Chong, S.; Chao, S.; Gullo-Brown, J.; Guo, P.; et al. Discovery of $(R)$-7-cyano-2,3,4,5-tetrahydro-1-(1H-imidazol-4ylmethyl)-3-(phenylmethyl)-4-(2-thienylsulfonyl)-1H-1,4-benzodiazepine (BMS-214662), a farnesyltransferase inhibitor with potent preclinical antitumor activity. J. Med. Chem. 2000, 43, 3587-3595.

33. Das, K.; Clark, A.D.; Lewi, P.J.; Heeres, J.; de Jonge, M.R.; Koymans, L.M.H.; Vinkers, H.M.; Daeyaert, F.; Ludovici, D.W.; Kukla, M.J.; et al. Roles of conformational and positional adaptability in structure-based design of TMC125-R165335 (etravirine) and related non-nucleoside reverse transcriptase inhibitors that are highly potent and effective against wild-type and drug-resistant HIV-1 variants. J. Med. Chem. 2004, 47, 2550-2560. 
34. Fleming, F.F.; Yao, L.H.; Ravikumar, P.C.; Funk, L.; Shook, B.C. Nitrile-containing pharmaceuticals: Efficacious roles of the nitrile pharmacophore. J. Med. Chem. 2010, 53, 7902-7917.

35. Wu, J.; Shi, Q.; Chen, Z.; He, M.; Jin, L.H.; Hu, D.Y. Synthesis and bioactivity of pyrazole acyl thiourea derivatives. Molecules 2012, 17, 5139-5150.

36. Kornienko, A.N.; Pil'o, S.G.; Kozachenko, A.P.; Prokopenko, V.M.; Rusanov, E.B.; Brovarets, V.S. Reaction of 2-aryl-4-cyano-1,3-oxazole-5-sulfonyl chlorides with 5-amino-1H-pyrazoles and 5-amino-1H-1,2,4-triazole. Chem. Heterocycl. Comp. 2014, 50, 76-86.

37. Thorson, J.S.; Hosted, T.J.; Jiang, J.; Biggins, J.B.; Ahlert, J. Natures carbohydrate chemists the enzymatic glycosylation of bioactive bacterial metabolites. Curr. Org. Chem. 2001, 5, 139-167.

38. Wu, G.; Robertson, D.H.; Brooks, C.L.; Vieth, M. Detailed analysis of grid-based molecular docking: A case study of CDOCKER-A CHARMm-based MD docking algorithm. J. Comput. Chem. 2003, $24,1549-1562$.

Sample Availability: Samples of the title compounds $\mathbf{3 a}-\mathbf{3 p}$ are available from the authors.

(C) 2015 by the authors; licensee MDPI, Basel, Switzerland. This article is an open access article distributed under the terms and conditions of the Creative Commons Attribution license (http://creativecommons.org/licenses/by/4.0/). 